\title{
FRACTIONAL MODEL OF A GASTROCNEMIUS MUSCLE FOR TETANUS PATTERN
}

\author{
Laurent Sommacal ${ }^{(1)}$, Pierre Melchior ${ }^{(1)}$, Jean-Marie Cabelguen ${ }^{(2)}$, Alain Oustaloup ${ }^{(1)}$ and Auke Jan ljspeert ${ }^{(3)}$ \\ ${ }^{(1)}$ LAPS - UMR 5131 CNRS \\ Université Bordeaux1 - ENSEIRB \\ 351 cours de la Libération \\ F33405 TALENCE cedex, France \\ Phone : +33 (0)540 002400 \\ Email: surname.name@laps.u- \\ bordeaux1.fr \\ (2)INSERM E 0358 \\ Institut Magendie, 1 rue Camille St Saëns \\ F33077 BORDEAUX cedex, FRANCE \\ Phone: +33 (0)557 574052 \\ Email: Jean- \\ marie.cabelguen@bordeaux.inserm.fr \\ ${ }^{(3)}$ EPFL, Swiss Federal Institute of \\ Technology \\ School of Computer and \\ Communication Sciences \\ IC-ISIM-LSL INN 241 \\ $\mathrm{CH}-1015$, LAUSANNE, Switzerland \\ Phone: (+41) 216932658 \\ Email: auke.ijspeert@epfl.ch
}

\section{ABSTRACT}

This study talks about gastrocnemius muscle identification. During biological activation, every contractile structure is unsynchronized. Likewise, contraction and relaxation phases depend on all contractile elements, the activation type and the state of health. Moreover, gastrocnemius muscle is composed of three fibre types: Fast Fatigable (FF), Fast Resistant (FR) and Slow (S) fibres.

Some recent works highlight a fractal structure of the muscle, which consolidate the approach based on the use of a non integer (or fractal) model to characterize its dynamic behavior. A fractional structure model, due to its infinite dimension nature, is particularly adapted to model complex systems with few parameters and to obtain a real time exploitable model.

According to its complexity, muscle structure and activation mechanisms, and to these previous considerations, an identification based on fractional model is presented.

A model is proposed for the tetanus pattern response in a high tiredness state. It is based on a multi-model structure, which corresponds to the decomposition in contraction and relaxation phases. This multi-model structure is expected to be included subsequently in agonist-antagonist structure.

\section{INTRODUCTION}

The importance of the striated muscles contraction in animal organism physiology is considerable. Indeed, these muscles are involved during partial or global organism moves (locomotion). The knowledge of the effective contribution of muscular contractions to locomotor activity, makes possible to associate cinematic changes to physiological (tiredness) or pathological origin modifications of the muscle fibre properties (myopathies).

The rhythmic and stereotyped nature of locomotor moves, as well as the conservation of several of their cinematic characteristics within vertebrate, including man, justifies studies that have been devoted to the striated muscles behavior during locomotion. Electromyographic recordings carried out on various species, permit to specify the muscular activations sequence during the locomotor cycle [13].

The contribution of muscular activations recorded for the observed locomotor movements remains nevertheless very badly known, in particular, due to the lack of data, concerning both the mechanical properties of muscles working under dynamic conditions and those coming from the locomotor apparatus (articulations, skin).

Thus, this paper aims for determining the muscular activation contribution to the locomotor movements for an 
inferior vertebrate (urodele) by means of a mathematical modeling of the muscular contraction in dynamic conditions. It is based on electromyographic and kinematic data obtained during a former study $[3,4]$.

The striated muscle structure is widely described in biology. Three fibre types [15] make up this muscle type. Myofilaments groups are the constituents of each fibre, which can be reduced to Actin and Myosin filaments. The fibre types present different characteristics, like contraction and relaxation delays and feeding types.

Some others recent works $[1,2,5,8,12]$ present a fractal structure of the muscle, which consolidate the approach based on the use of non integer (or fractal) model to characterize its dynamic behaviour. A fractional structure model, due to its infinite dimension nature, is particularly adapted to model complex systems with few parameters, and to obtain real time exploitable model: muscle complexity can be described thanks to fractional models, with a model containing a reduced number of parameters.

After this introduction, Section 2 explains the fractional derivative. In Section 3, the time domain system identification by fractional model is summarized. The experimental protocol is detailed in section 4 . Then, section 5 presents the muscular response to tetanus pattern identification. Finally, a conclusion is given in Section 6.

\section{FRACTIONAL DERIVATIVE}

\section{Definition of fractional calculus}

For a fractional derivative operator, output $s(t)$ is the $n^{\text {th }}$ derivative of input $e(t)$ :

$s(t)=D^{n} e(t)$,

where $D=d / d t$ is the derivative operator and $n$ is no longer limited to being an integer $[10,11,14]$ : it can be also a fractional, real, imaginary or complex number.

When the real part of the fractional order is negative, it is in fact a fractional integral operator which is also defined by (1). So, we can refer to fractional derivative or fractional integral indifferently.

The fractional integral concept appeared in the $19^{\text {th }}$ century with Laplace, Liouville, Abel and Riemann [7]. Riemann's definition is:

$D^{-n} f(t)=\frac{1}{\Gamma(n)} \int_{c}^{t}(t-\theta)^{n-1} f(\theta) \mathrm{d} \theta$,

where $c$ is the integral reference, $\Gamma(n)$ the gamma function, and $n$ the fractional integration order. Unfortunately, for historical reasons, the word fractional is used in the literature. It is however understood as meaning any real, imaginary, or complex number.

\section{Discrete form: Grünwald's definition}

The first derivative of a function is defined by [6]:

$\mathrm{D} f(t)=\lim _{h \rightarrow 0} \frac{f(t)-f(t-h)}{h}$

Introducing the left finite difference $\Delta_{h} f(t)$, namely:

$\Delta_{h} f(t)=f(t)-f(t-h)$,

or $\Delta_{h} f(t)=\left(1-q^{-1}\right) f(t)$,

where $q^{-1}$ is the delay operator, and $h$ the finite sampling step, the effective computation of ( 3 ) is defined by:

$\mathrm{D} f(t) \approx \frac{1-q^{-1}}{h} f(t)$.

If a function $f(x)$ is differentiable up to order $n$, expression (6) can be extended:

$\mathrm{D}_{h}^{n} f(t) \approx\left(\frac{1-q^{-1}}{h}\right)^{n} f(t)$.

Expression (7) can be used to define a fractional derivative by direct replacing $n$ by a fractional order. It is one more time understood that $n$ can be any real, imaginary, or complex number.

A series expansion of (7) leads to:

$D^{n} f(t) \approx \frac{1}{h^{n}}\left(\sum_{k=0}^{\infty}(-1)^{k} a_{k}(n) q^{-k}\right) f(t)$,

where $a_{k}(n)$ are the binomial coefficients:

$a_{k}(n)=\frac{\Gamma(n+1)}{\Gamma(k+1) \Gamma(n-k+1)}$

So, the fractional differentiation of a function will be computed using Grünwald's definition:

$D^{n} f(t) \approx \frac{1}{h^{n}} \sum_{k=0}^{\infty}(-1)^{k} a_{k}(n) f(t-k h)$.

This becomes the same as the Riemann-Liouville fractional integral when the sampling interval tends towards zero. A negative real part for the fractional order of differentiation $n$ is chosen for (10), so the fractional integral (2) can be computed. Definition (10) shows that the fractional integral of a function at instant $t$ takes into account the function values at all instants of its past: $f(t), f(t-h), f(t-2 h)$, $f(t-3 h)$ etc., which are samples of its history. 


\section{TIME DOMAIN SYSTEM IDENTIFICATION BY FRACTIONAL MODEL}

The parametric estimation method used results from the output error method extension to non integer order. The objective consists in estimating, based on the input and output data measured on the system to identify, the differential equation coefficients and orders (real or fractional):

$$
\begin{aligned}
& a_{1} D^{n_{1}} y(t)+a_{2} D^{n_{2}} y(t)+\ldots+a_{L} D^{n_{L}} y(t)= \\
& b_{1} D^{m_{1}} u(t)+b_{2} D^{m_{2}} u(t)+\ldots+b_{Q} D^{m_{Q}} u(t)
\end{aligned}
$$

The ARX structure presented Figure 1, can be deduced:

$$
y(s)=\frac{b_{1} s^{m_{1}}+b_{2} s^{m_{2}}+\ldots+b_{q} s^{m_{Q}}}{a_{1} s^{n_{1}}+a_{2} s^{n_{2}}+\ldots+a_{L} s^{n_{L}}} u(s) .
$$

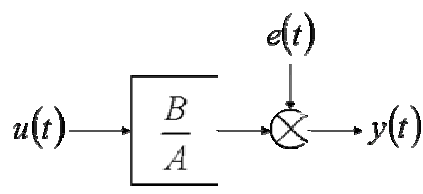

Figure 1: ARX structure used

$\mathrm{A}$ and $\mathrm{B}$ are polynomial for $s$, with condition $n_{L} \geq m_{Q}$

$A(s)$ is applied on $y(s)$ and $B(s)$ on $u(s)$ in frequency domain

$$
\sum_{l=1}^{L} a_{l} s^{n_{l}} y(s)=\sum_{q=1}^{Q} b_{q} s^{m_{q}} u(s),
$$

and in time domain, is equivalent to:

$$
\sum_{l=1}^{L} a_{l} \frac{d^{n_{l}}}{d t^{n_{l}}} y(t)=\sum_{q=1}^{Q} b_{q} \frac{d^{m_{q}}}{d t^{m_{q}}} u(s) .
$$

This equation and Grünwald's definition allow to obtain the ARX time response for a fractional model:

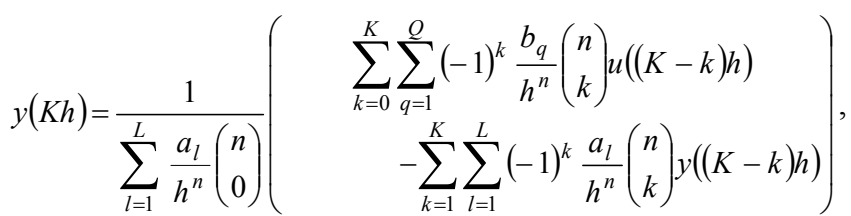

where the coefficients,

$$
a_{1}, \ldots, a_{L}, n_{1}, \ldots, n_{L}, b_{1}, \ldots, b_{Q}, m_{1}, \ldots, m_{Q}
$$

have to be estimated.
The simulated response obtained with this model is then compared to those of real system, which is noised (figure 2).

The cost function, $\varepsilon=y^{*}(t)-\hat{y}(t)$, orientates the choice of parameters (16) to become minimum. This orientation is based on the nonlinear simplex algorithm [16, 17]. This allows estimating orders and coefficients of (12).

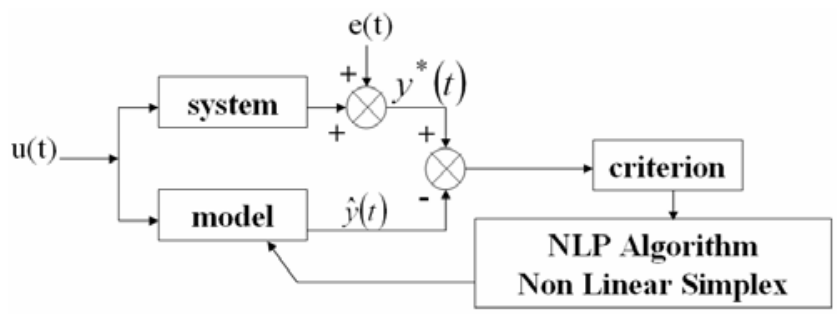

Figure 2: Output error model

\section{EXPERIMENTAL PROTOCOL}

\section{Experimental and schematic protocol}

Experiments about this project are performed on Rana esculenta frog muscles, and more particularly on the gastrocnemius one figure 3 . The structure and the composition of this muscle are identical to those of salamanders, with which next experiments will be conducted. The frog muscle advantage is a bigger length, easier to experiment. The aim is to model muscle behavior thanks to tetanus, swimming and walking salamander cycles

Frogs are demedulated and decerebrated to eliminate arc-back. Legs skin is removed. Exposed muscles and skin are covered with some paper moisted with Ringer solution. Control electrodes are applied on sciatic nerve, between the pelvis and the gastrocmenius. The tendon between the gastrocnemius and the ankle is severed and fixed on an isometric sensor (Phymep UF-1). Experimentally, the muscle length is the in situ length measured in the median posture. Pulses are sent on the muscle thanks to the sciatic nerve. The pulse time width is $1 \mathrm{~ms}$. The amplitude is fixed, during a test phase beginning every experiment, to obtain maximum amplitude. In this way, each fibre is excited. The isometric sensor allows us to translate the muscular tension into a potential variation, which is displayed on an oscilloscope. Electric signals (pulses for the stimulation and the contraction responses) are recorded thanks to an $\mathrm{A} / \mathrm{N}$ converter (CED 1401) and the software Spike 2. Data are received as text files. The sampling frequency for excitation and isometric responses is $10 \mathrm{kHz}$. Data text files will be used for the muscle identification.

The figure 4 displays the muscular response to a regularly spaced pulses pattern. In these conditions, one can observe an amplitude decrease and a smooth modification of the muscular contraction dynamic behavior. 

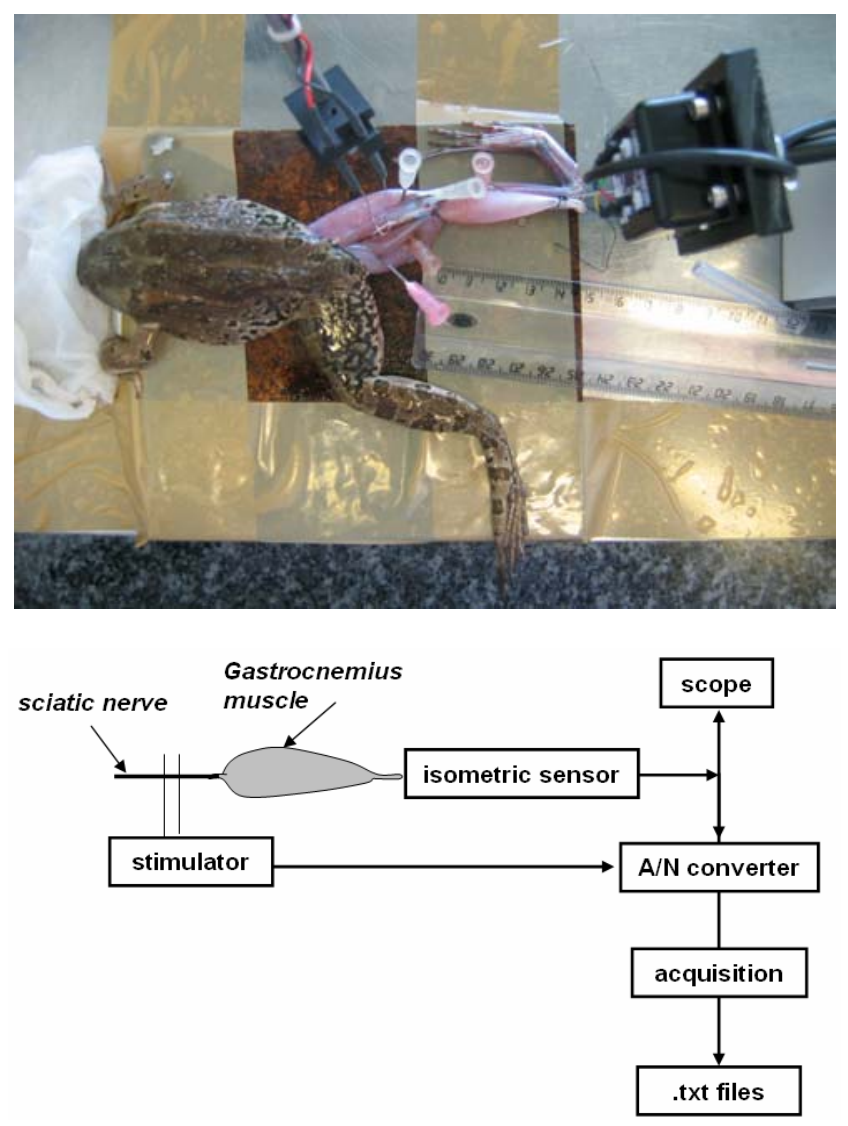

Figure 3: Experimental and schematic protocol



Figure 4: Amplitude variation of muscular responses for regularly spaced pulses pattern

Eight pattern sequences are applied on muscle to obtain a predictable and representative behavior. For each pattern, different regularly spaced pulses sequences are applied. Figure 5 shows these sequences ( 74 pulses applied on muscle). Each response is studied in order to identify the muscular behavior.

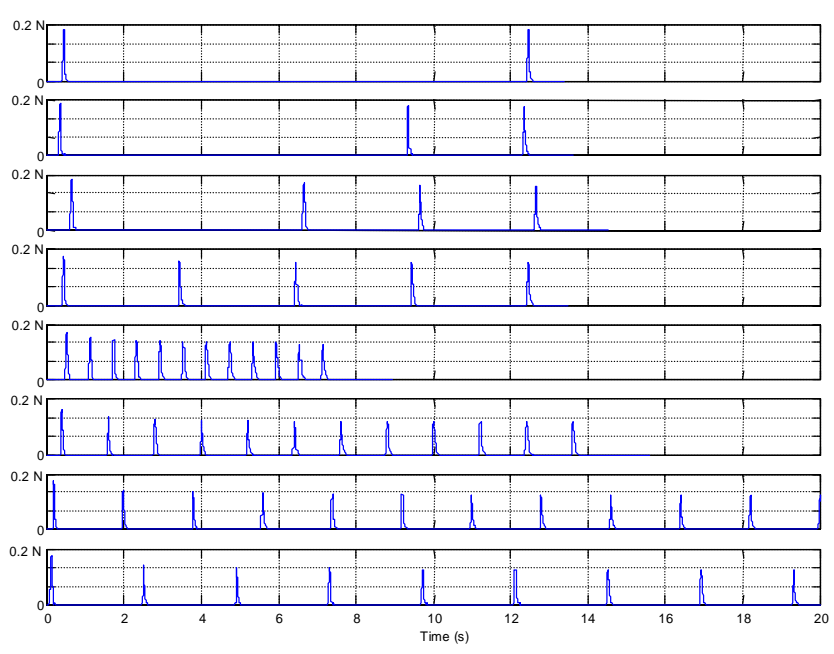

Figure 5: Muscular responses to 8 sequences with different regularly spaced pulses patterns

\section{Single pulse model}

An integer model is obtained thanks to parametric estimation so as to study the muscle behavior. A minimum amount of 11 parameters is adopted to have a good modeling:

$$
\begin{aligned}
& y(t)+1.210^{-1} D^{1} y(t)+4.110^{-3} D^{2} y(t)+6.710^{-5} D^{3} y(t) \\
& +5.710^{-7} D^{4} y(t)+2.810^{-9} D^{5} y(t)-6.910^{-11} D^{6} y(t) \\
& =1.410^{-3} u(t)+3.110^{-2} D^{1} u(t)+2.110^{-3} D^{2} u(t) \\
& -2.610^{-6} D^{3} u(t)+8.810^{-8} D^{4} u(t) .
\end{aligned}
$$

with $u(t)$, the signal applied on sciatic nerve and $y(t)$, the muscular strength response.

To test the ability of the non integer tool to report this behavior, a same model structure based on fractional orders is defined. As we have no particular knowledge about the derivative orders, the half integer order step is chosen (0.5), which is the extreme non integer case.

$$
\begin{aligned}
& y(t)-2.610^{-1} D^{0.5} y(t)+9.010^{-2} D^{1} y(t)-1.410^{-2} D^{1.5} y(t) \\
& +1.910^{-3} D^{2} y(t)-1.310^{-4} D^{2.5} y(t)+6.810^{-6} D^{3} y(t) \\
& =-1.510^{-2} u(t)+2.610^{-2} D^{0.5} u(t)+1.410^{-2} D^{1} u(t) \\
& -1.810^{-3} D^{1.5} u(t)+2.610^{-4} D^{2} u(t) .
\end{aligned}
$$

The figure 6 represents one of 74 muscular responses, the integer and fractional model responses.

In both cases, the structure models, obtained using a parametric estimation, allow to obtain a muscular response with a very small error, in either of integer or fractional cases. 




Figure 6: Muscular response to a pulse (solid), integer (dotted) and fractional models (dashed)

\section{A model for the amplitude response}

For isolated pulses, muscular responses show an amplitude variation. It appears also few dynamic variations. To have a global modeling to a pulse pattern, a mean response model is determined after the normalization of each response. Amplitude variations are described thanks to a second model, function of the delay between two successive pulses,

The figure 7 shows the normalized mean response with the fractional model response. The maximum and minimum muscular responses are also shown on this figure. The identification is applied on this normalized mean response.

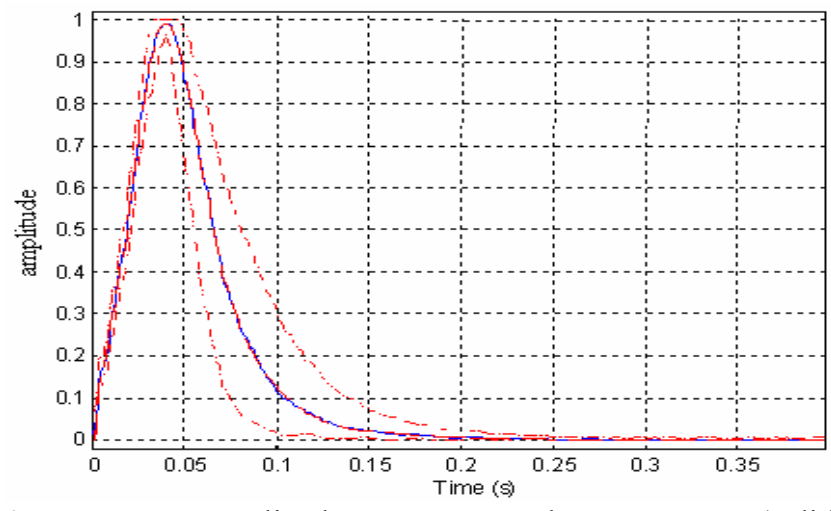

Figure 7: Normalized mean muscular response (solid), fractional model response (dotted) and minimum/maximum experiment responses (dashed)

The obtained model is the following one, with 11 fractional parameters:

$$
\begin{aligned}
& y(t)-1.810^{-1} D^{0.5} y(t)+7.010^{-2} D^{1} y(t)-9.110^{-3} D^{1.5} y(t) \\
& \quad+1.410^{-3} D^{2} y(t)-8.810^{-5} D^{2.5} y(t)+5.010^{-6} D^{3} y(t) \\
& =-1.310^{-2} u(t)+2.010^{-2} D^{0.5} u(t)+4.210^{-2} D^{1} u(t) \\
& -5.010^{-3} D^{1.5} u(t)+2.710^{-4} D^{2} u(t) .
\end{aligned}
$$

The behavior model is computed from the average of normalized responses, depending on time elapsed between two successive pulses.
The figure 8 shows amplitude development for eight experiment sequences. When the pattern is reduced to sufficiently near pulses, the maximum muscular amplitude can be reduced to a decreasing exponential function and can be modeled by:

$A(t)=(1-K) \mathrm{e}^{-t / \tau}+K, \quad$ with $K \approx 0.7$ and $\tau \approx 2.5 s$.

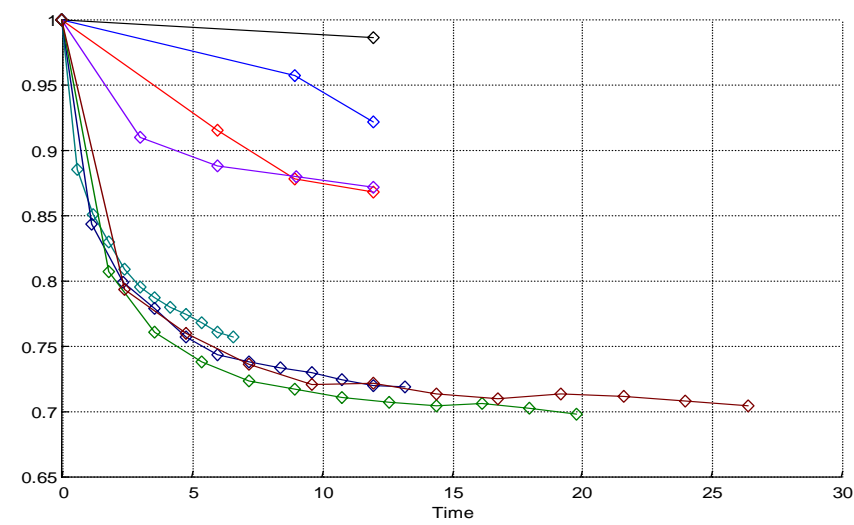

Figure 8: Amplitude development depends on the delay between two successive pulses

So, (18) associated with (19), allows to get a global model to closely-spaced pulses, where (18) reports dynamic and (19) the amplitude variation.

\section{MUSCULAR RESPONSE TO THE TETANUS PATTERN IDENTIFICATION}

\section{Tetanus pattern determination}

In a second stage, the obtained model will be used with swimming and walking patterns, to verify the model validity. EMG data obtained on in vivo salamander during swimming cycle, as seen on figure 9, do not seem to be reproducible. The patterns resulting from the application of a threshold on these biological data, a second time, prove these patterns are not yet reproducible.

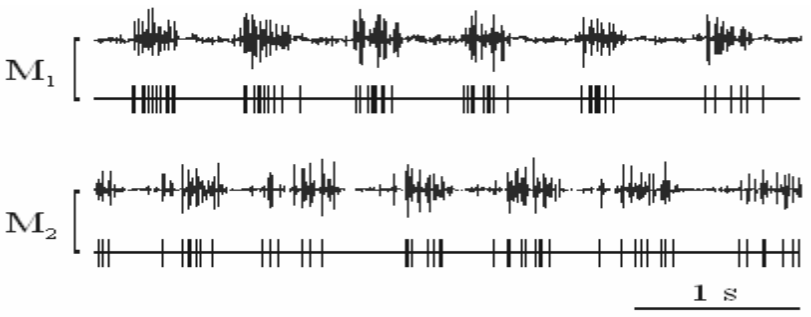

Figure 9: Activation of 2 antagonist myomeres $\left(\mathrm{M}_{1}\right.$ and $\left.\mathrm{M}_{2}\right)$ during swimming cycles for the pleurodele. For each myomere, the plot above represents the electromyographic activity (EMG) and the plot below is the pulse train, after EMG threshold.

The tetanus pattern is easier to build, because it makes possible the creation of an artificial pattern which allows to have the minimum rising time and a maximal amplitude 
response. Firstly, the tetanus pattern is studied and secondly, the obtained model is going to be ratified by means of its pattern (18).

To create this pattern, which implicates the muscle tetanus, the pulses arrangement acts as a substitute for EMG signals propagated on each nerve axons during in vivo conditions. The main advantage is this pattern is totally reproducible, since every fibre is activated by pattern. This response pattern results from an iterative pulse addition presented figure 10. The time fixed between two pulses corresponds to the biggest response amplitude and the smallest twitch time. The delay between each exercise is sufficient to let the muscle in the same tiredness state, $15 \mathrm{~s}$ minimum.
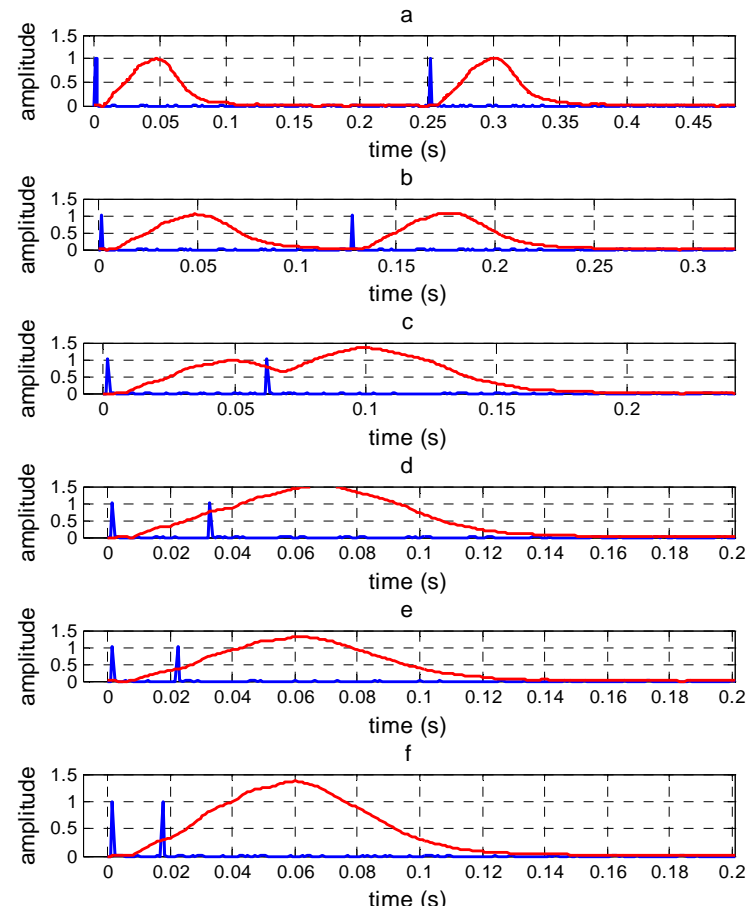

Figure 10: Amplitude of muscular responses for 5 delays between two pulses: $250 \mathrm{~ms}$ (a), $125 \mathrm{~ms}$ (b), $60 \mathrm{~ms}$ (c), $30 \mathrm{~ms}$ (d) and $15 \mathrm{~ms}(\mathrm{e})$

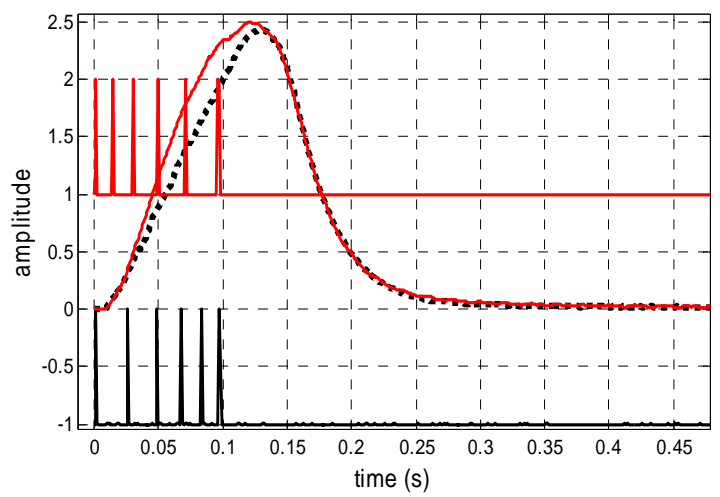

Figure 11: Response for the decreasing time gap pattern (dashed) and for the increasing time gap pattern (solid). The rising time is smaller in the second case
In the case of 6 pulses, the optimal response is obtained with the following pattern: 24, 21, 18, 15, $12 \mathrm{~ms}$. Nevertheless, EMG data show the global signal is richer at the beginning than at the end. So, the figure 11 displays the response stemming from the pattern above determined and the response stemming from the inverse pattern. Both amplitudes are quite equal but the peak response time is smaller with the second pattern.

Due to this result, a second study has allowed to create the following global pattern, for which, the twitch time is minimized with a maximum amplitude: $12,12,16,16,16,16$, $18,18,18,18, \ldots \mathrm{ms}$

This pattern, defined by these time gaps, is adopted as the tetanus pattern.

\section{Identification of the muscular response from the tetanus pattern}

A typical tetanus pattern response is presented in the

Figure 12. The time domain identification of this response has been done.

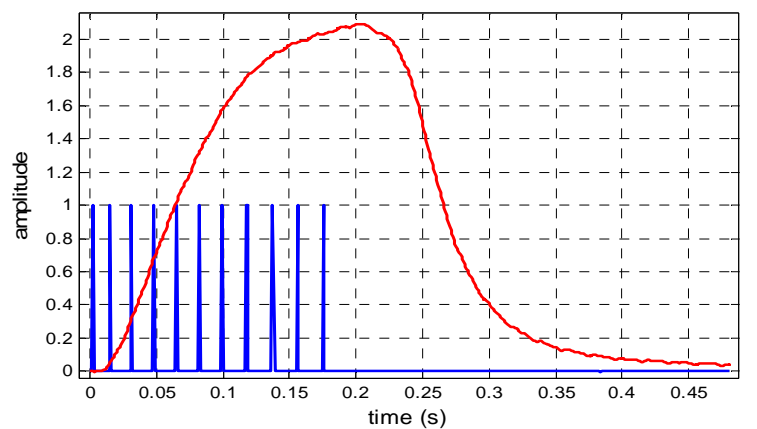

Figure 12: One typical muscular response obtained with the tetanus pattern

In a first step, the tetanus pattern is applied to the models (17) and (18) corresponding to an isolated pulse response, compared to the muscular response obtained with the tetanus pattern (figure 13). The difference between the experimental response and the models response proves these models are not sufficient to explain all tetanus response.

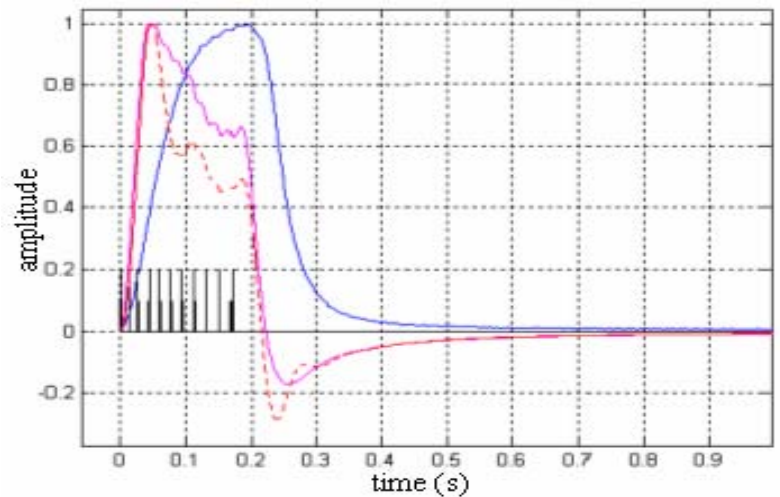

Figure 13: Comparison of the integer (light, solid), fractional (dotted) models responses corresponding to an isolated pulse response with the muscular responses (dark, solid), to the tetanus pattern (black) 
So, in a second step, another approach consists to try to identify the global muscular response obtained with the tetanus pattern (figure 14). The fractional model size is 15 parameters. The difference between the experimental response and the model response proves that a global model is not sufficient to explain all tetanus response anew.
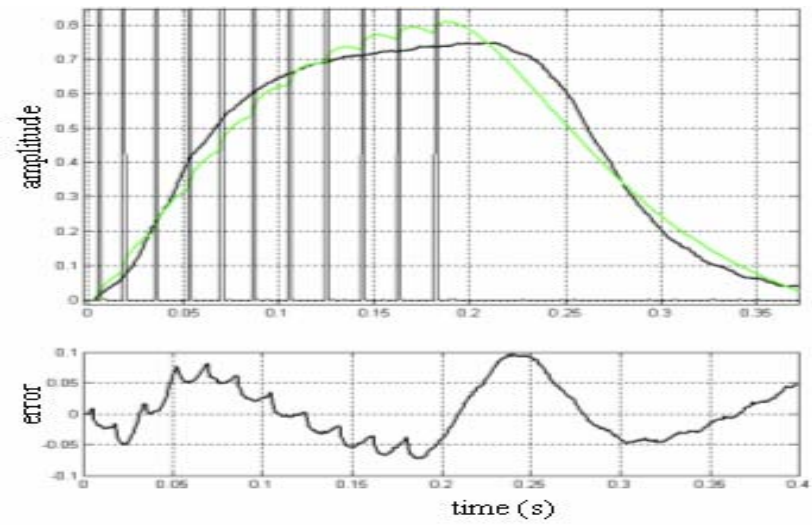

Figure 14: Comparison of the global model response (light) with the muscular response (black) to the tetanus pattern and the error

Thus, to approach the biological phenomenon of twitch and relaxing muscular phases, in a third step, a new approach is defined: a multi-model structure [9] has been tested. The twitch and relaxing phases are treated separately (figure 15), due to the fact biological literature mentions a physical mechanisms difference [15]. This induces a non linear behavior, which can be first approximated with a linear multimodel. This response corresponds to a tired muscle response.

Because the muscle and nerve frequency bands are low, the signal input can not be rich enough in frequencies to allow a reliable frequency-domain system identification.

The twitch model (figure 16) is designed form the twitch phase of response, which corresponds to the eleven first pulses of tetanus pattern. The figure 17 presents the relaxation phase.

The optimization algorithm is based on a fractional order model, which includes by nature integer orders. Only the structure size is fixed, orders and coefficients are free. The algorithm optimizes both coefficients and orders, and converges toward fractional model with 6 parameters:

\section{Twitch phase:}

$G_{T}(s)=\frac{1}{s}\left(\frac{44.27}{s^{1.23}+40.66}\right)+\frac{1}{s}\left(\frac{-51.13}{s^{1.38}+52.98}\right)$,

Relaxation phase:

$G_{R}(s)=\left(\frac{192.66859}{s^{0.86389984}+6.2722972}\right)+\left(\frac{1766.43}{s^{1.13}+62.50}\right)$.

Since it is a global identification, these parameters (coefficients and orders) have no physiological meaning.

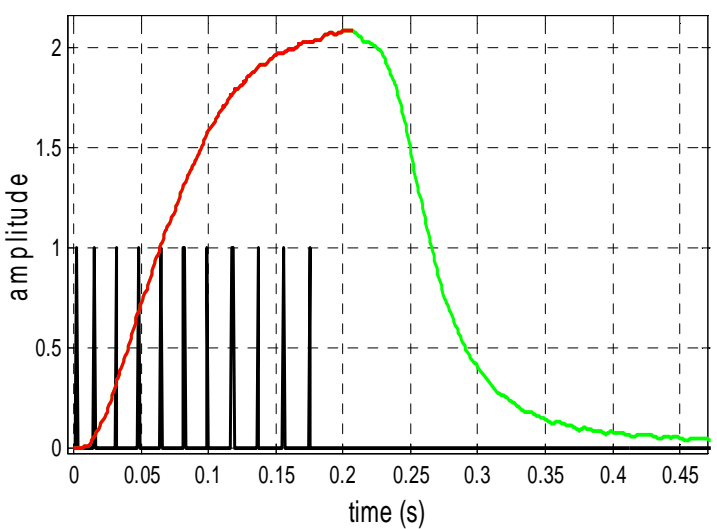

Figure 15: Twitch (dark) and relaxation response phase (light) for tetanus pattern (black)

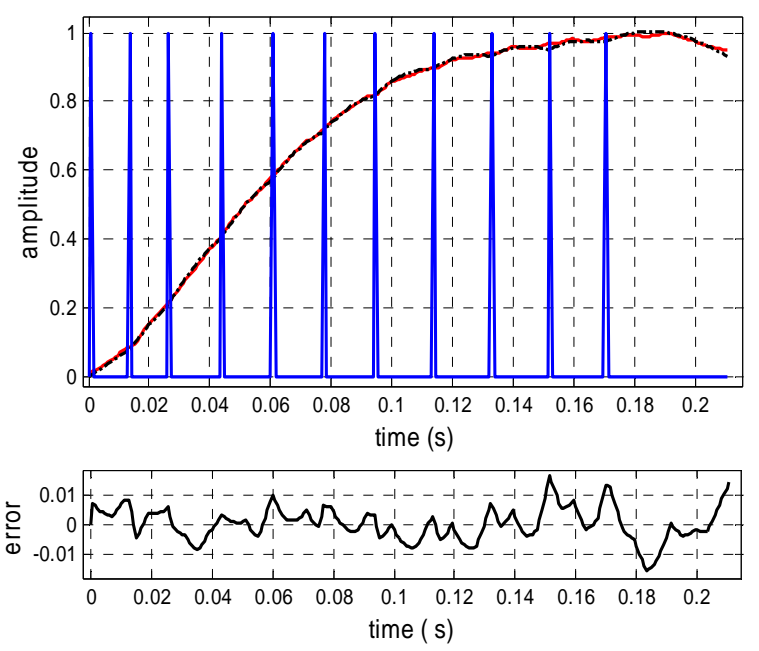

Figure 16: Twitch phase of muscular response (solid) and twitch fractional model (black, dotted) for tetanus pattern and error
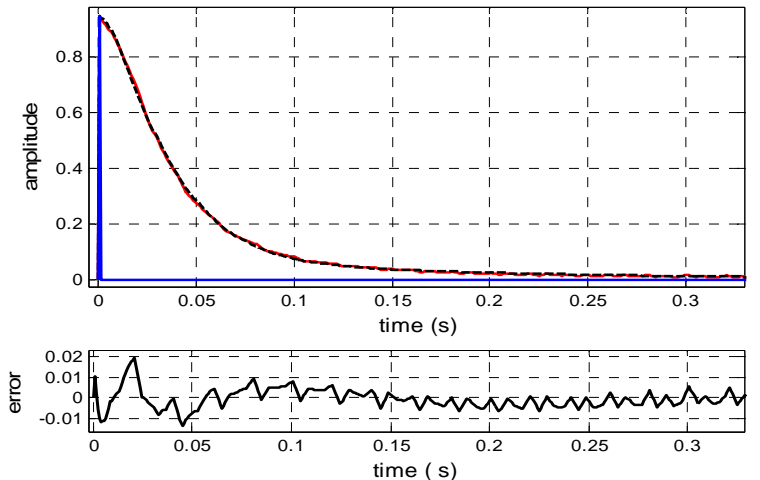

Figure 17: Relaxation phase of muscular response (solid) and relaxation fractional model (black, dashed) and error

The validation phase has been tested respectively with the sixth, seventh and eleventh pulses of tetanus pattern. 

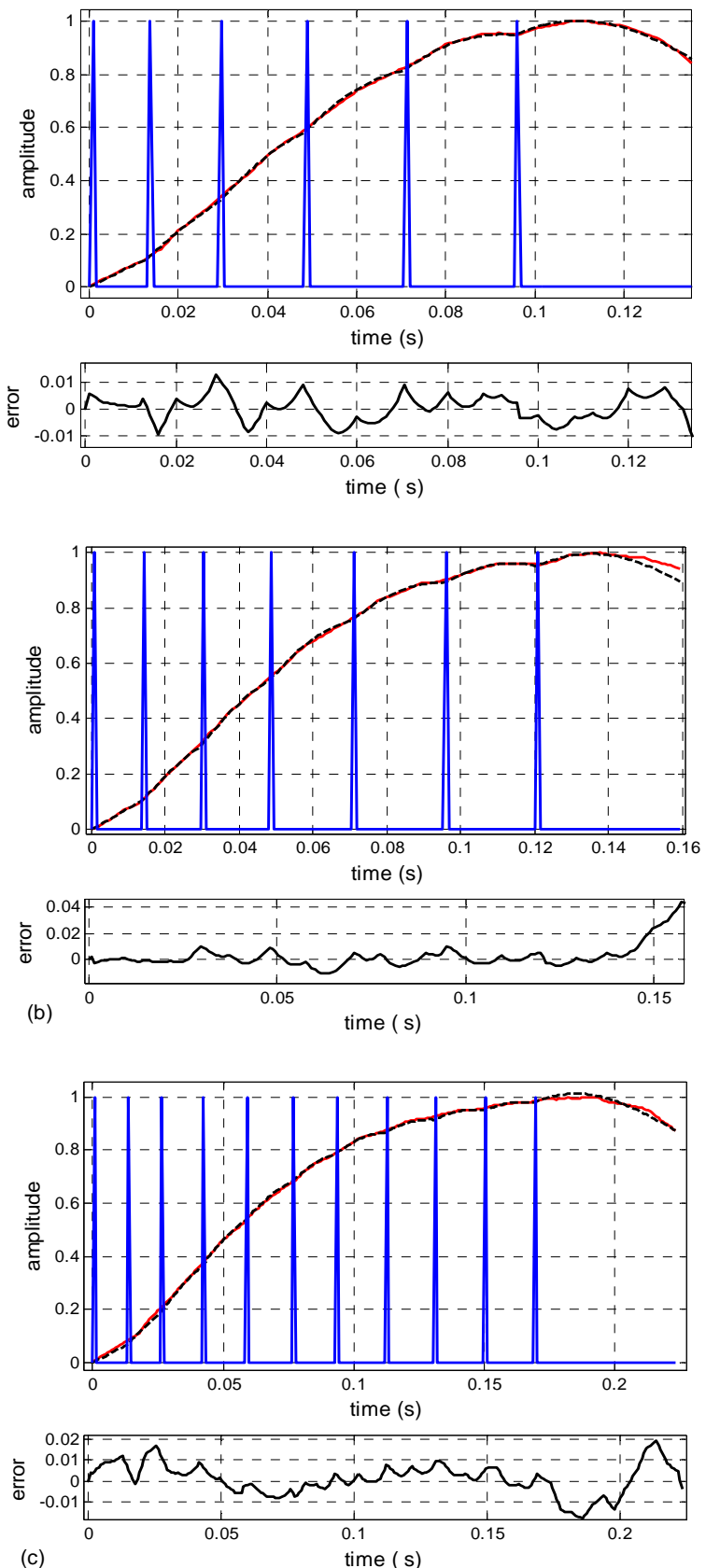

Figure 18: Validation phase: comparison of the twitch phase model responses (black, dotted) with the muscular response (solid) respectively to the 6 (a), 7 (b) and 11 (c) first pulses of tetanus pattern and errors

The figure 18 presents the comparison of the twitch phase model responses with the muscular response respectively to the 6 (a), 7 (b) and 11 (c) first pulses of tetanus pattern. In the different cases, the obtained model allows a good reconstruction of the twitch phase.
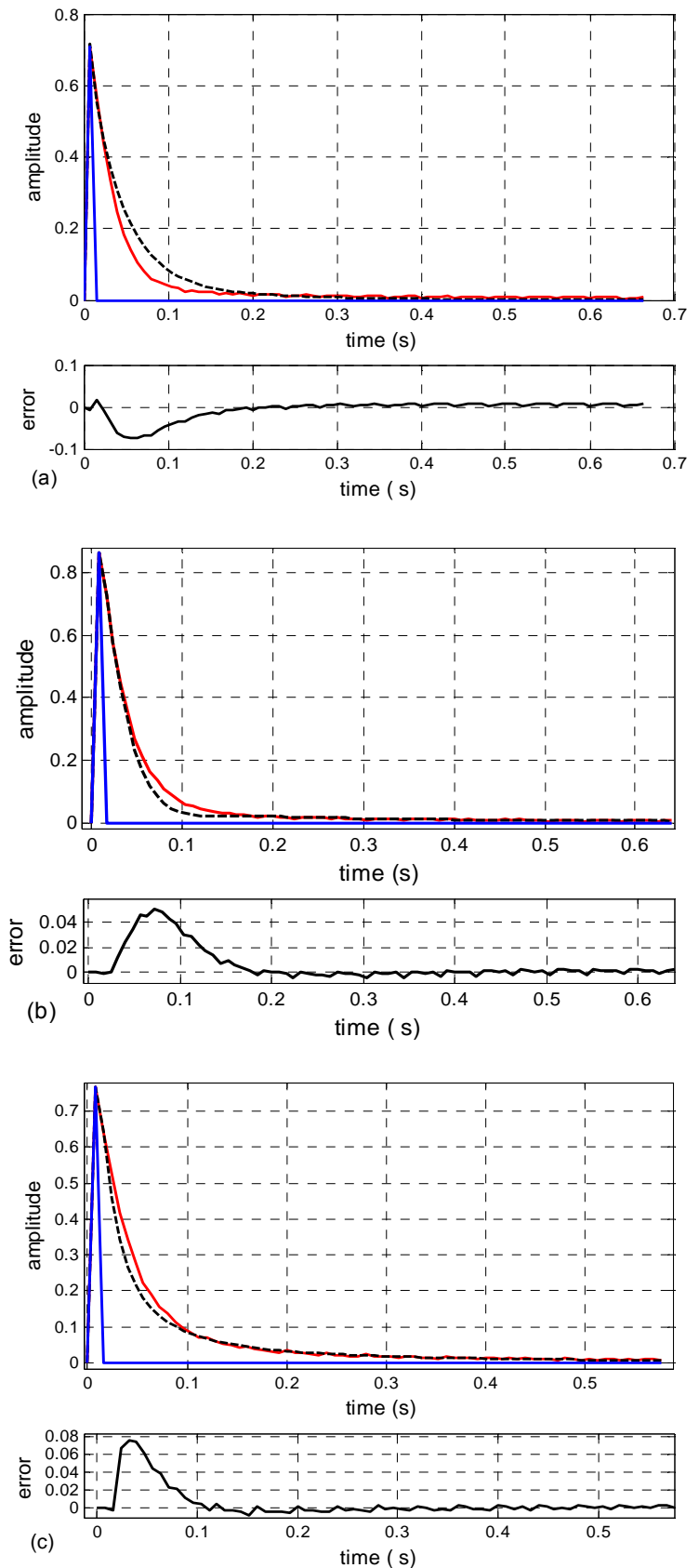

Figure 19: Validation phase: comparison of the relaxation phase model responses (black, dotted) with the muscular response (solid) respectively to the 6 (a), 7 (b) and 11 (c) first pulses of tetanus pattern and errors

The figure 19 presents the comparison of the relaxation phase model responses with the muscular response respectively to the 6 (a), 7 (b) and 11 (c) first pulses of tetanus pattern. For relaxation phase identification, the obtained model allows also a good reconstruction, but the error is worse than twitch phase identification one. Moreover, the error is not centered on zero, which is significant of a bias error. 


\section{CONCLUSION}

This paper introduces the gastrocnemius muscle identification by fractional model.

A first model is proposed for the muscular response to a single pulse. The muscular response from the tetanus pattern is also studied. An artificial global tetanus pattern has been determined. A fractional model of the gastrocnemius muscle response to tetanus pattern is proposed. It is based on a multimodel structure, which corresponds to the decomposition in contraction and relaxation phases, according to physical phenomenon described in biological literature.

The optimization algorithm considers fractional order model, which includes by nature integer orders. Only the structure size is fixed, orders and coefficients are let free. The algorithm optimized both coefficients and orders, and converges toward fractional model with 6 parameters.

The validation phase shows the comparison of the model responses with the muscular response for different sizes of tetanus pattern. In the different cases, obtained models allow a good reconstruction of the twitch phase, but the existence of a bias error in the relaxation phase.

These models are obtained with a particular muscle tiredness state. Also, it is necessary to study the tiredness to obtain a model which can predict all tetanus response.

\section{ACKNOWLEDGEMENTS}

This work is done within the framework of the ACI "Neurosciences Intégratives et Computationnelles", Project NIC0010: "Locomotion in salamander: neural control, mathematical and neuro-mechanical modelling". The authors gratefully acknowledge the financial support of the French Research Secretary.

\section{REFERENCES}

[1] Arsos G. A., Dimitriu P. P., 2004, "A fractal characterization of the type II fibre distribution in the extensor digitorum longus and soleus muscles of the adult rat", Muscle \& Nerve, John Wiley \& sons, Inc., New York, $\mathrm{N}^{\circ} 18$, pages 961-968.

[2] Cross S. S., 1997, "Fractals in pathology", Journal of Pathology, John Wiley \& sons, Inc., New York, Vol. 182, 1-8.

[3] Delvolvé I., Bem T., Cabelguen J.-M., 1997, "Epaxial and limb muscle activy during swimming and terrestrial stepping in the adult newt, Pleurodeles waltl", J. Neurophysiol., 78, 638650 .
[4] Delvolvé I., Branchereau P., Dubuc R. and Cabelguen J.M., 1999, "Fictive patterns of rhythmic motor activity induced by NMDA in an in vitro brainstem-spinal cord preparation from an adult urodele amphibian", J. Neurophysiol., 82, 10741078.

[5] Goldberger A. L., Amaral L. A. N., Hausdorff J. M., Ivanov P.Ch., Peng C.-K., Stanley H. E., February 2002, "Fractal dynamics in physiology: Alterations with disease and aging", PNAS, Vol. 99, Suppl. 1, pages 2466-2472.

[6] Grünwald A. K., 1867, "Ueber begrenzte Derivationen und deren Anwendung", Z Angew. Math. Phys., 12, pages 441-480.

[7] Liouville.J., 1832, "Mémoire sur le calcul des différentielles à indices quelconques", Ecole Polytechnique,13, n²1, pages 71-162.

[8] Lowen S. B., Cash S. S., Poo M.-M., Teich M. C. August 1997, "Quantal Neurotransmitter Secretion Rate Exhibits Fractal Behavior", the Journal of Neuroscience, 17 (15), pages 5666-5677.

[9] Malti R., Aoun M., Battaglia J.-L., Oustaloup A., Madami K., August 1989, "Fractional Multimodels - Application to heat transfert modelling", $13^{\text {th }}$ IFAC Symposium on System Identification, Rotterdam, the Netherlands.

[10] Miller, K.S., Ross, B., 1993, "An Introduction To The Fractional Calculus and Fractional Differential Equation", John Wiley \& Sons, Inc., New York.

[11] Oustaloup, A., 1995, "La Derivation Non Entière", Hermes, Paris.

[12] Ravier P., Buttelli O., Couratier P., 2005, "An EMG fractal indicator having different sensitivities to changes in force and muscle fatigue during voluntary static muscle contractions", Electromyogr Kinesiol, 15(2): 210-221.

[13] Rossignol S., "Neural control of stereotypic limb movements", Int. Handbook of Physiology - Rowell L.B. and Sheperd J.T. Editions, American Physiological Society, 173216, 1996.

[14] Samko, A. G., Kilbas, A. A., Marichev, O. I., 1987, "Fractional Integrals and Derivatives", Gordon and Breach Science, Minsk.

[15] Shepherd, G. M.., 1994, "Neurobiology", Oxford, New york.

[16] Subrahmanyam M. B., August 1989, "An Extension of the Simplex Method to Constrained Nonlinear Optimization", Int. Journal of Optimization Theory and Applications, Vol. 62, no. 2, pages 311-319.

[17] Woods D. J.,May 1985, "An interactive Approach for solving Multi-Objective Optimization Problems", Technical Report 85-5, Rice University, Houston. 